'Departamento de Especialidades Médicas, Facultad de Medicina, Universidad de La Frontera, Temuco-Chile.

2Unidad de Neurología, Hospital Dr. Hernán Henríquez Aravena, Temuco-Chile.

${ }^{3}$ Centro de Investigación en Epidemiología Cardiovascular y Nutricional (EPICYN), Universidad de La Frontera, Temuco-Chile.

${ }^{4}$ Departamento de Salud Pública, Facultad de Medicina, Universidad de La Frontera. Temuco-Chile.

${ }^{a}$ Nutricionista, MSc. bDoctorando en Salud Pública. Universidad Pública de Navarra (UPNA), Pamplona, España.

Trabajo sin fuente de financiamiento.

Recibido el 20 de octubre de 2016, aceptado el 27 de abril de 2017.

Correspondencia a: Álvaro Soto Venegas Departamento de Especialidades Médicas,

Facultad de Medicina, Universidad de La Frontera. Francisco Salazar \# 01145 , Temuco-Chile. alvaro.soto@ufrontera.cl

\section{Evolución del protocolo de trombolisis endovenosa en ataque cerebrovascular isquémico agudo}

\author{
ÁLVARO SOTO V. ${ }^{1,2,3}$, GLADYS MORALES I.,4,a,b, \\ MARCELA GRANDJEAN B. ${ }^{1,2}$, DÉBORA POLLAK W..$^{1,2}$, \\ CAROLINA DEL CASTILLO C. ${ }^{1,2}$, PÍA GARCÍA F. ${ }^{1,2}$, \\ ALEXIS VON JOHNN A. ${ }^{1,2}$, ALFONSO RIQUELME G. ${ }^{1,2}$
}

\section{Intravenous thrombolysis for acute ischemic stroke. A four years' experience in a Chilean public hospital}

Background: Intravenous thrombolysis (IVT) with alteplase (tissue plasminogen activator) is the standard pharmacological treatment in acute ischemic stroke (AIS), reducing disability in patients. Aim: To report the results a thrombolysis protocol during four years in a regional public hospital. Material and Methods: Data from 106 consecutive patients aged $68 \pm 13$ years (57\% men) who were treated with IVT, from May 2012 until April 2016, was analyzed. Results: The median door-to-needle time was 80 minutes (interquartile range $=57-113$ ). The median National Institute of Health Stroke Scale (NIHSS) scores on admission and at discharge were was 11.5 and 5 points respectively. At discharge, 27\% of hospitalized patients had a favorable outcome $(n=99)$, defined as having 0 to 1 points in the modified Rankin scale. Symptomatic intracerebral hemorrhage and mortality rates were 5.7 and $13.1 \%$, respectively. The thrombolysis rate rose from $0.7 \%$ in 2012 to $6 \%$ in 2016. Conclusions: The implementation of 24/7 neurology shifts in the Emergency Department allowed us to increase the amount and quality of IVT in our hospital, as measured by the rate of thrombolysis and by process indicators such as door-to-needle time.

(Rev Med Chile 2017; 145: 468-475)

Key words: Fibrinolytic Therapy; Stroke; Time-to-Treatment; Thrombolytic Therapy; Tissue Plasminogen Activator.
E n Chile, las enfermedades cerebrovasculares (ECV) son la principal causa de muerte, con una tasa de 50,6 defunciones por 100.000 habitantes el año $2011^{1}$. Además, son la primera causa específica de años de vida ajustados por discapacidad (AVISA) en mayores de 74 años y la quinta entre 60 y 74 años ${ }^{1}$. La tasa de incidencia de ECV es de 130 por 100 mil habitantes/año ${ }^{2,3}$. El Ataque Cerebrovascular Isquémico (ACVi) es la causa más frecuente de ECV en Chile representando aproximadamente $65 \%$ de todos los eventos cerebrovasculares ${ }^{2,3}$. La tasa de incidencia de ECV, calculada como diagnóstico de egreso hospitalario, en el período 2001-2010, en el Servicio de Salud Araucanía Sur, fue de 961,3 por 100 mil habitantes/año ${ }^{4}$. A su vez la Novena Región presenta la tasa más alta de mortalidad por ECV del país ${ }^{5}$ El ACVi es el motivo de consulta neurológica más frecuente en el Servicio de Urgencia Adultos (SUA) del Hospital Dr. Hernán Henríquez Aravena (HHHA) de Temuco, dando cuenta del 30,7\% de las atenciones realizadas por el neurólogo ${ }^{6}$. La trombolisis 
endovenosa (TEV) con alteplase (activador del plasminógeno tisular), administrada hasta $4,5 \mathrm{~h}$ desde el inicio de los síntomas, es el tratamiento farmacológico de referencia en el ACVi agudo, reduciendo la probabilidad de dependencia de los pacientes ${ }^{7,8}$. Desde 1996, la TEV constituye el estándar de manejo, del ACVi agudo en los países desarrollados. Si bien la implementación de la TEV fue rápida en el Sistema Privado de Salud chileno $^{9}$, recién el año 2011, fue comunicada la primera experiencia de un protocolo de TEV en un hospital público del país ${ }^{10}$. Desde mayo de 2012 se realiza TEV en el HHHA, que cuenta con neurólogos presenciales, en modalidad 24/7, en el Servicio de Urgencia desde julio de $2013^{11}$. Nuestro establecimiento cuenta con 730 camas, es el único Hospital de Alta Complejidad de la Novena Región, y atiende a una población beneficiaria de aproximadamente 800 mil habitantes. La Unidad de Neurología del HHHA no cuenta con un servicio propio, y depende del Servicio de Medicina Interna. Nuestro hospital carece de una Unidad de Tratamiento del Ataque Cerebral (UTAC).

El objetivo de este estudio es describir la evolución de nuestro protocolo de TEV, particularmente en relación al número de pacientes tratados por año y al comportamiento de los indicadores de proceso de la intervención, como el tiempo puerta-aguja (TPA).

\section{Material y Método}

Serie de pacientes consecutivos que recibieron TEV, entre mayo de 2012 y abril de 2016 . El protocolo de TEV se basó en los estudios NINDS (1995) y ECASS III (2008), es decir, trombolisis dentro de $3 \mathrm{~h}$, y entre 3 y $4,5 \mathrm{~h}$, respectivamente. Además se realizaron modificaciones considerando la literatura médica más reciente $e^{12}$ y la experiencia local, particularmente en relación a la edad (mayores de 80 años) y la pertinencia de algunas contraindicaciones relativas ${ }^{13,14}$. En la Tabla 1 se exponen los criterios de inclusión, exclusión y precaución del protocolo de TEV.

La estimación de la severidad del compromiso neurológico se realizó mediante la escala National Institute of Health Stroke Scale (NIHSS) que comprende 42 puntos, donde un aumento de puntaje indica mayor compromiso neurológico ${ }^{15}$. Se practicó una tomografía axial computada (TAC)

\section{Tabla 1. Criterios de inclusión, exclusión y precaución para Trombolisis Endovenosa}

\section{Criterios de Inclusión}

Síntomas neurológicos por un período inferior a 4,5 h, con hora de inicio definida

Déficit neurológico medible por la escala NIHSSa

Tomografía computarizada de cerebro sin signos de hemorragia intracraneal

Consentimiento informado firmado

\section{Criterios de exclusión}

Ataque Cerebrovascular isquémico dentro de los últimos 3 meses

Traumatismo encéfalocraneano o cirugía del sistema nervioso central en los últimos 3 meses

Infarto agudo al miocardio dentro de los últimos 21 días

Cirugía mayor en los últimos 14 días o biopsia de órgano

Antecedentes de hemorragia intracraneana

Antecedentes de coagulopatía (hemofilia, von Willebrand)

Hemorragia gastrointestinal o vía urinaria en los últimos 21 días

Uso de anticoagulantes orales con INR ${ }^{b}>1,7$ o nuevos anticoagulantes en las últimas $48 \mathrm{~h}$

Crisis convulsiva al inicio de los síntomas (excepto que difusión confirme infarto)

Punción arterial en sitio no compresible en los últimos 7 días

Punción lumbar en los últimos 7 días

Embarazo o parto durante el último mes

Antecedentes conocidos de neoplasia con riesgo de sangrado

PAc sistólica > $185 \mathrm{mmHg}$ y/o PA diastólica > $110 \mathrm{mmHg}$ refractaria a hipotensores endovenosos

Evidencia de sangrado sistémico

Sospecha de embolia séptica o endocarditis infecciosa

Signos neurológicos rápidamente regresivos

NIHSS $<4$

Glicemia $<50$ mg/dl o $>400 \mathrm{mg} / \mathrm{dl}$

Prolongación de TTPK ${ }^{\mathrm{d}}>40 \%$

Recuento de Plaquetas $<100.000 / \mathrm{mm}^{3}$

Hematocrito $<25 \%$

Tiempo de protrombina $>15 \mathrm{seg}$

Hemorragia o transformación hemorrágica precoz

Infarto extenso constituido

\section{Criterios de precaución}

Edad mayor a 80 años

Déficit neurológico severo (NIHSS > 22 ptos)

Signos precoces de infarto extenso (ASPECTSe $<7$ )

aNational Institute of Health Stroke Scale; ' $/$ International Nor-

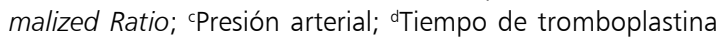
parcial activada; ' ${ }^{A}$ Alberta Stroke Program Early CT Scan Score. 
de cerebro, al ingreso, en todos los pacientes, con el objetivo de descartar una hemorragia intracraneal y otros diagnósticos. La TEV con alteplase fue administrada en dosis de $0,9 \mathrm{mg} / \mathrm{kg}$, con un máximo de $90 \mathrm{mg}$. El 10\% se administra en bolo durante 1 min y el 90\% restante en una infusión por $60 \mathrm{~min}$. Los pacientes fueron trombolisados en el Box de Reanimación del SUA. Se obtuvo la firma del consentimiento informado en todos los casos. Una vez terminada la TEV, los pacientes, en su gran mayoría, fueron trasladados a la Unidad de Tratamiento Intermedio (UTI) para completar un período de observación de $48 \mathrm{~h}$. En caso, de no observarse complicaciones, los pacientes fueron hospitalizados en el Servicio de Medicina Interna para neuroprotección, rehabilitación y realización del estudio etiológico. Siete sujetos fueron trasladados precozmente a clínicas privadas de Temuco, por lo que fueron excluidos de la evaluación funcional al alta.

En todos los casos se estimaron los tiempos de atención, que constituyen indicadores de proceso, medidos en minutos: tiempo inicio-puerta, medido desde el inicio de los síntomas hasta la llegada al SUA; tiempo puerta-TAC desde el ingreso al SU hasta la realización del scanner de cerebro; tiempo puerta-aguja, tomado desde ingreso al SUA y el bolo de alteplase; y el tiempo inicio-aguja, medido desde el inicio del cuadro hasta el inicio de la TEV.

Los ACVi fueron catalogados clínicamente según la clasificación de Oxfordshire ${ }^{16}$, en infartos: totales de circulación anterior (TACI), parciales de circulación anterior (PACI), lacunares (LACI) y de circulación posterior (POCI). Los pacientes fueron evaluados mediante un scanner de cerebro sin contraste, 24 h post trombolisis, o en caso de deterioro neurológico, definido como un aumento de 4 o más puntos en la escala NIHSS. Se utilizó la escala Alberta Stroke Program Early CT Scan (ASPECTS) para identificar cambios isquémicos tempranos ${ }^{17}$. Además, se identificaron las hemorragias intracerebrales (HIC), clasificándolas según los criterios del estudio SITS-MOST ${ }^{18}$. Las hemorragias intracerebrales sintomáticas (HICs) fueron definidas como una hemorragia local o remota visualizada en TAC realizada hasta 7 días posterior a la TEV, asociada a un deterioro neurológico, siendo la principal causa determinante del deterioro neurológico o muerte ${ }^{19}$ (criterio ECASS II). La monitorización clínica, durante la hospitalización, se realizó con la escala NIHSS. Los
99 pacientes hospitalizados en el HHHA fueron evaluados al alta con la escala de Rankin modifica$\mathrm{da}(\mathrm{mRS})^{20}$, que corresponde a una medición de la discapacidad, y con la escala NIHSS. Los puntajes mRS varían desde 0 puntos (paciente asintomático), y aumenta según el grado de discapacidad hasta 6 puntos (fallecimiento).

Según su causa, los ACVi fueron clasificados según los criterios de TOAST en 5 grupos: ateromatosis de grandes arterias, cardioembólicos, oclusión de vasos pequeños, disección arterial y causa no determinada ${ }^{21}$.

Se estimó la tasa anual de trombolisis endovenosa para el período 2012-2016. El análisis estadístico se efectuó con el software Stata 14.0. Las variables continuas se describen con medidas de tendencia central y dispersión: promedio \pm desviación estándar (DE) y/o medianas con rangos intercuartiles (RIQ).

\section{Resultados}

Ciento seis pacientes fueron tratados en el período. Siete pacientes recibieron TEV durante el período de neurología, en horario hábil ( 8 a 18 h), el resto de los procedimientos se efectuaron bajo la modalidad 24/7. El promedio de edad de los pacientes fue 67,7 años $(\mathrm{DE}=12,6)$, con una mediana de 69 años. El 56,6\% de los pacientes fueron varones $(n=60)$. Las características clínicas de los pacientes trombolisados se exponen en la Tabla 2, y sus factores de riesgo en la Tabla 3.

Respecto a los parámetros de tiempo, destaca una mediana del tiempo inicio-puerta de 93,5 min (RIQ 60-134). La mediana del tiempo puerta-aguja (TPA) fue de $80 \mathrm{~min}$ (RIQ 57-113). La mediana del tiempo inicio-aguja fue de 190 min (RIQ 154235). La mediana de la Escala NIHSS al ingreso fue 11,5 puntos (RIQ 7-17). En 30 pacientes (28,3\%) el TPA aguja fue menor a $60 \mathrm{~min}$. Asimismo se observó una constante disminución de los TPA y un progresivo aumento del porcentaje de pacientes tratados en menos de 60 min desde su ingreso a urgencia (Tabla 4). En 46 pacientes $(43,4 \%)$ la trombolisis se inició dentro de $3 \mathrm{~h}$ de evolución del ACVi.

En la clasificación clínica de los ACVi, según los criterios de Oxfordshire destaca 44,3\% de infartos TACI; 35,9\% de infartos PACI; 15,1\% de infartos POCI y $4,7 \%$ de infartos LACI. Las etiologías de 
Tabla 2. Características clínicas de los pacientes trombolisados

\begin{tabular}{|lc|}
\hline Características & $\begin{array}{c}\text { Pacientes } \\
(\mathbf{n}=\mathbf{1 0 6})\end{array}$ \\
\hline Edad (media $+\mathrm{DE}^{\mathrm{a}}$ ) & $67,7 \pm 12,6$ \\
\hline Género masculino (\%) & $60(56,6)$ \\
\hline Etnia mapuche (\%) & $6(5,7)$ \\
\hline ASPECTS (mediana, RIQc) & $9(7-10)$ \\
\hline NIHSS ${ }^{\text {d }}$ pretrombolisis & $12 \pm 6,5$ \\
\hline NIHSS postrombolisis & $10 \pm 6,5$ \\
\hline PAe sistólica (mmHg) & $152 \pm 23,4$ \\
\hline PA diastólica (mmHg) & $82 \pm 15,1$ \\
\hline Glicemia (mediana, RIQ) & $124(103-150)$ \\
\hline Dosis alteplase (mg) & $66,6 \pm 14,4$ \\
\hline
\end{tabular}

aDesviación estándar; ${ }^{b}$ Alberta Stroke Program Early CT Scan Score; 'Rango intercuartil; 'National Institute of Health Stroke Scale; ePresión arterial.
Tabla 3. Factores de riesgo de los pacientes trombolisados

\begin{tabular}{|lcc|}
\hline Factor de riesgo & $\mathbf{n}$ & $\mathbf{\%}$ \\
\hline Hipertensión arterial & 83 & 78,3 \\
\hline Diabetes mellitus & 33 & 31,1 \\
\hline Dislipidemia & 24 & 22,6 \\
\hline Tabaquismo & 14 & 13,2 \\
\hline Ex fumador & 7 & 6,6 \\
\hline ACV ${ }^{\text {a }}$ previo a 3 meses & 12 & 11,3 \\
\hline ACV dentro de 3 meses & 1 & 0,9 \\
\hline AlT ${ }^{b}$ & 2 & 1,9 \\
\hline Fibrilación auricular & 33 & 31,1 \\
\hline Insuficiencia cardiaca & 12 & 11,3 \\
\hline Alcoholismo & 3 & 2,9 \\
\hline
\end{tabular}

${ }^{a}$ Ataque cerebrovascular; ${ }^{b}$ Ataque isquémico transitorio.

Tabla 4. Evolución de la tasa de trombolisis, parámetros de tiempo y porcentaje de pacientes que recibió trombolisis en menos de $60 \mathrm{~min}$

\begin{tabular}{|ccccccc|}
\hline Año & $\mathbf{n}$ & $\begin{array}{c}\text { Tasa TEV } \\
(\mathbf{\%})\end{array}$ & $\begin{array}{c}\text { Mediana TIPb } \\
\text { (min) }\end{array}$ & $\begin{array}{c}\text { Mediana TPAc } \\
\text { (min) }\end{array}$ & $\begin{array}{c}\text { Mediana TIAd } \\
\text { (min) }\end{array}$ & \% TPA < 60 min \\
\hline 2012 & 5 & 0,7 & 24 & 80 & 154 & 0 \\
\hline 2013 & 11 & 1,2 & 93 & 111 & 200 & 36,4 \\
\hline 2014 & 24 & 2 & 79 & 97 & 180,5 & 8,3 \\
\hline 2015 & 44 & 3,6 & 91 & 71 & 182,5 & 40,1 \\
\hline 2016 & 22 & 6 & 132,5 & 67 & 220 & 27,7 \\
\hline Total & 106 & 2,3 & 93,5 & 80 & 190 & 28,3 \\
\hline
\end{tabular}

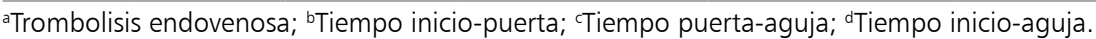

los ACVi, según la clasificación de TOAST, es la siguiente: $23,4 \%$ de causa aterotrombótica; $35,9 \%$ de causa cardioembólica; $5,7 \%$ por disección arterial; $5,7 \%$ de infartos lacunares y $29,3 \%$ de causa indeterminada.

Para los 99 pacientes que completaron su hospitalización en el HHHA, la mediana de hospitalización fue de 8 días (RIQ 5-13). Respecto al resultado funcional de la intervención medido con la Escala Modificada de Rankin (mRS) al alta (Figura $1)$, destaca que 27 pacientes $(27,3 \%)$ egresaron sin discapacidad $(\mathrm{mRS}=0-1)$. La mediana de la escala NIHSS al alta fue de 5 puntos (RIQ 1-14). La tasa de mortalidad fue de $13,1 \%$. En relación a las causas de fallecimiento, en 7 pacientes se debió al ACVi. 2 casos por transformación hemorrágica sintomática y en 4 pacientes por complicaciones no relacionadas al ACVi: cáncer bronquial, sepsis de foco cutáneo, sepsis de foco pulmonar y neumonía grave. Además se registraron 13 pacientes con transformación hemorrágica $(12,3 \%)$, de las cuales $6(5,7 \%)$ fueron sintomáticas.

Cuatro pacientes desarrollaron un ACVi intrahospitalario, con un sujeto de 66 años que sufrió el infarto cerebral posterior a la realización de una coronariografía. Por otra parte, en 4 pacientes se realizó TEV más trombectomía mecánica. En este subgrupo, un paciente de 51 años falleció como 


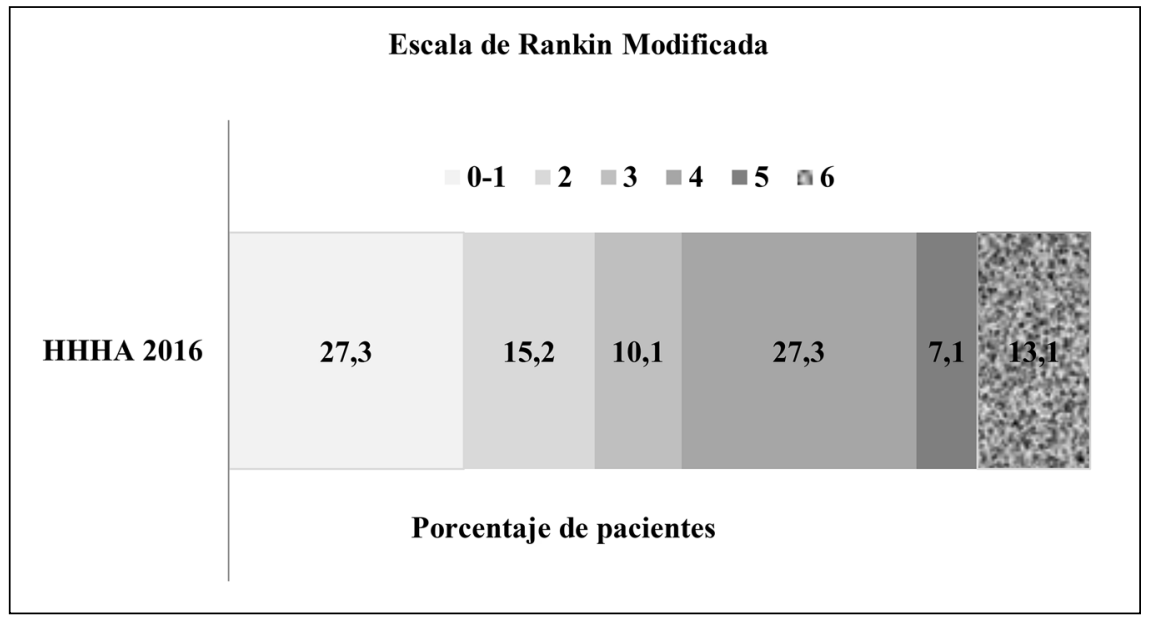

Figura 1. Evaluación de la discapacidad al alta en pacientes con ACV isquémico que recibieron trombolisis endovenosa. consecuencia de un infarto maligno de arteria cerebral media derecha debido a una disección carotídea oclusiva.

Entre mayo de 2012 y abril de 2016 se diagnosticaron 4503 ACVi en nuestro hospital, con un promedio de 1126 casos/año. La tasa de trombolisis aumentó de 0,7\% el año 2012 a 6\% el año 2016. En la Tabla 4 se detalla la evolución del número de pacientes tratados por año, del TPA y del porcentaje de los sujetos que fueron trombolisados dentro de $60 \mathrm{~min}$.

\section{Discusión}

El ACVi es la urgencia neurológica más frecuente en nuestro hospital, dando cuenta del 30\% de las atenciones realizadas por el neurólogo en el SUA ${ }^{6}$. Por otra parte, la eficacia y seguridad de la trombolisis endovenosa, en el ACVi, ha sido establecida en ensayos clínicos y estudios observacionales desde hace más de 20 años. La eficacia de la TEV es altamente tiempo-dependiente, cuanto antes se instaure el tratamiento, es mejor el desenlace funcional ${ }^{22,23}$. La guía norteamericana de manejo del ACVi agudo recomienda un tiempo puerta-aguja $\leq 60 \mathrm{~min}^{24}$. Se ha comunicado que esta recomendación se cumple en menos del 30\% de los pacientes que reciben TEV ${ }^{25}$. En esa misma línea, en un registro que incluyó 45079 TEV, la mediana del TPA fue de $67 \mathrm{~min}^{26}$. En ese contexto, consideramos que los indicadores de proceso, como el tiempo puerta-aguja, son fundamentales para la evaluación de la calidad de la atención en el ACVi agudo, y en particular, de la TEV. La reducción del TPA permite disminuir el tiempo inicio-aguja. Se ha estimado que por cada $15 \mathrm{~min}$ de disminución del retraso para la trombolisis se obtiene $4 \%$ de aumento en la probabilidad de un buen desenlace funcional ${ }^{23}$. En nuestra serie la mediana del TPA fue de $80 \mathrm{~min}$. Las posibles causas para el resultado de este indicador son: servicio de urgencia colapsado, retraso en la evaluación del priorizador, retraso en la evaluación por neurólogo, retraso en la toma de neuroimágenes, falta de cupo en el box de reanimación, espera de resultado de exámenes (INR), etc.

En nuestra serie no fue posible realizar la evaluación de la discapacidad de los sujetos a los 3 meses. En este sentido se han utilizado evaluaciones de resultado precoces como el puntaje en la escala NIHSS al segundo y al séptimo día de evolución del ACVi, que han mostrado predecir con una adecuada precisión resultados funcionales a los 3 meses $^{27,28}$. En nuestro estudio la mediana de la escala NIHSS al alta fue de 5 puntos, con un NIHSS pre trombolisis de 11,5 puntos. La mayor severidad de los ACVi, comparada con otras series, podría ser explicada por el alto porcentaje de infartos tipo TACI y de infartos de causa cardioembólica. El 5,7\% de hemorragias intracerebrales sintomáticas (HICs) observado en nuestra serie es comparable a lo comunicado en estudios nacionales y extranjeros. Por ejemplo, un reciente meta-análisis de 12 ensayos clínicos aleatorizados de TEV hasta $6 \mathrm{~h}$ desde el inicio de 
Tabla 5. Comparación de los resultados obtenidos en trombolisis endovenosa en 3 hospitales públicos chilenos

\begin{tabular}{|c|c|c|c|}
\hline Indicadores & HHHA $^{a}$ & HSJD $^{b}$ & HBLc \\
\hline $\mathrm{n}$ pacientes & 106 & 54 & 19 \\
\hline TPA (promedio) & 89 & 87 & 111 \\
\hline HIC sintomática & $5,7 \%$ & $5,5 \%$ & $0 \%$ \\
\hline Mortalidad & $13,1 \%$ & $14,8 \%$ & $5,3 \%$ \\
\hline Tasa de trombolisis & $6 \%$ & $3,8 \%$ & $4,9 \%$ \\
\hline
\end{tabular}

aHospital Dr. Hernán Henríquez Aravena; ${ }^{\circ}$ Hospital San Juan

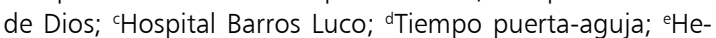
morragia intracerebral.

los síntomas, que incluyó 7.012 pacientes, reportó una tasa de HICs de 7,7\%, con una tasa de HICs fatal durante los primeros 7 días de 3,6\%, y una mortalidad de $8,9 \%$ dentro de 7 días, y de 19,1\% hasta el final del período de seguimiento ${ }^{29}$. Al presentar nuestros resultados debemos recalcar que el HHHA no dispone de una infraestructura específica para atender a pacientes neurológicos, es decir, una Unidad de Tratamiento del Ataque Cerebral (UTAC). Estas unidades han demostrado su costo-efectividad para disminuir la mortalidad y discapacidad por $\mathrm{ACV}^{30}$. En nuestra realidad no todos los pacientes trombolisados acceden a la UTI y completan 24-48 h de observación en el SUA, siendo posteriormente hospitalizados en el Servicio de Medicina Interna.

Las experiencias reportadas de TEV en hospitales públicos chilenos son escasas. En el 2011, Figueroa y cols. comunicaron en los primeros 19 casos de pacientes trombolisados en un centro hospitalario público, $63 \%$ de pacientes en mRS 0-1 a los 3 meses, una mortalidad de 5,2\% y una tasa de trombolisis de $4,9 \%{ }^{10}$. En un artículo reciente, Guevara y cols. comunicaron una serie de 54 pacientes que recibieron TEV, en otro hospital público de Santiago ${ }^{31}$. Los resultados fueron $66,4 \%$ de mRS $0-1$ a los 3 meses, con $5,5 \%$ de HICs y una tasa de trombolisis de 3,8\%. La Tabla 5 compara nuestra serie con la experiencia de estos dos hospitales.

La tasa de trombolisis que partió en 0,7\% para el período mayo-diciembre 2012, se elevó a $6 \%$ en el período enero-abril 2016 (Tabla 4). Para este cambio consideramos que ha sido fundamental la presencia del neurólogo, desde julio 2013, en el Servicio de Urgencia en la modalidad 24/7. Nuestra tasa de trombolisis es comparable a la observada en otros hospitales públicos chilenos, pero es muy baja comparada con clínicas privadas nacionales que han comunicado una tasa mayor a $10 \%{ }^{1}$. Según nuestros registros, $19,1 \%$ de los pacientes con ACVi consultan dentro de $3 \mathrm{~h}$ de iniciado el cuadro, con una mediana de $10 \mathrm{~h}$ aproximadamente, hasta la consulta. Creemos que con campañas educativas dirigidas a la comunidad, $y$ la socialización de nuestro protocolo de TEV a los centros hospitalarios y de salud de la Novena Región, podemos aumentar la tasa de trombolisis a mediano plazo. Los pacientes trombolisados tuvieron una menor duración de su hospitalización (mediana de 8 días), considerando que la estadía promedio de pacientes que no reciben TEV ha sido estimada entre 14 y 15 días, en otros hospitales públicos $^{31,32}$.

Nuestros resultados, que constituyen la mayor serie reportada de TEV en ACVi agudo, en hospitales públicos chilenos, nos llenan de satisfacción y optimismo. Además constituyen un enorme aliciente para seguir aumentando el número de pacientes tratados y continuar mejorando la calidad de la atención. La TEV en ACVi agudo es factible de ser realizada en hospitales públicos, y particularmente en regiones de nuestro país. La presencia de neurólogos 24/7 en el Servicio de Urgencia nos ha permitido aumentar la cantidad y la calidad de la TEV en nuestro hospital, esto medido por tasa de trombolisis y mediante indicadores de proceso como el tiempo puerta-aguja.

\section{Referencias}

1. Ministerio de Salud de Chile. Plan de Acción Ataque Cerebrovascular, 2a Edición, 2014,http://www.worldstrokecampaign.org/component/rsform/?task=submissions.view.file\&hash $=\mathrm{b} 037 \mathrm{fb} 17712 \mathrm{dfb} 1 \mathrm{a} 88763$ eeafcle55 de\&Itemid $=232$.

2. Ministerio de Salud de Chile. Guía clínica AUGE. Accidente Cerebrovascular Isquémico en personas de 15 años y más. Serie de las guías clínicas de MINSAL, 2013,http://web.minsal.cl/portal/url/ item/7222754637e58646e04001011f014e64.pdf.

3. Lavados PM, Sacks C, Prina L, Escobar A, Tossi C, Araya $\mathrm{F}$, et al. Incidence, 30-day case-fatality rate, and prognosis of stroke in Iquique, Chile: a 2-year community-based prospective study (PISCIS project). Lancet 365 (9478): 2206-15. 
4. Doussoulin A, Rivas R, Sabelle C. Egresos hospitalarios por enfermedad cerebrovascular en el período 20012010 en el Servicio de Salud Araucanía Sur. Rev Med Chile 2016; 144: 571-6.

5. Lavados PM, Díaz D, Jadue L, Olavarría VV, Cárcamo DA, Delgado I. Socioeconomic and Cardiovascular Variables Explaining Regional Variations in Stroke Mortality in Chile: An Ecological Study. Neuroepidemiology 2011; 37: 45-51.

6. Soto A, Morales G, Pollak D, Jara V. Análisis de las consultas neurológicas en el Servicio de Urgencia de un hospital terciario. Rev Chil Neuro-Psiquiat 2016; 54 (2): 93-101.

7. National Institute of Neurological Disorders and Stroke rt-PA Stroke Study Group. Tissue plasminogen activator for acute ischemic stroke. N Engl J Med 1995; 333: 15817.

8. Hacke W, Kaste M, Bluhm E, Brozman M, Dávalos A, Guidetti D, et al. Thrombolysis with Alteplase 3 to 4.5 Hours after Acute Ischemic Stroke. N Engl J Med 2008; 359 (13): 1317-29.

9. Feuerhake W, Chamorro H, Araya F. Activador del plasminógeno tisular intravenoso en el tratamiento del infarto cerebral agudo. Rev Med Chile 1999; 127: 814-9.

10. Figueroa-Reyes T, Sáez MD, Mansilla LE, Sánchez VR, Nogales-Gaete J, Delgado BI. Experiencia de Trombolisis sistematizada en infarto cerebral agudo en un hospital público de Chile. Rev Med Chile 2011; 139: 1118-27.

11. Soto A, Pollak D, Von Johnn A, Grandjean M, Riquelme A, García P, et al. Thrombolysis with alteplase in acute ischaemic stroke at Dr. Hernan Henriquez Hospital of Temuco-Chile. Póster WFN15-1543 Stroke. XXII World Congress of Neurology. Santiago, Chile, October 31 November 5, 2015.

12. The IST-3 collaborative group. The benefits and harms of intravenous thrombolysis with recombinant tissue plasminogen activator within $6 \mathrm{~h}$ of acute ischaemic stroke (the third international stroke trial [IST-3]): a randomised controlled trial. Lancet 2012; 379: 2352-63.

13. Demaerschalk BM, Kleindorfer DO, Adeoye OM, Demchuk AM, Fugate JE, Grotta JC, et al; on behalf of the American Heart Association Stroke Council and Council on Epidemiology and Prevention. Scientific rationale for the inclusion and exclusion criteria for intravenous alteplase in acute ischemic stroke: a statement for healthcare professionals from the American Heart Association/American Stroke Association. Stroke 2015; STR.0000000000000086, published online before print December 22, 2015.

14. Balami JS, Hadley G, Sutherland BA, Karbalai H, Buchan AM. The exact science of stroke thrombolysis and the quiet art of patient selection. Brain 2013: 136; 352853.

15. Brott T, Adams HP Jr, Olinger CP, Marler JR, Barsan WG, Biller J, et al. Measurements of acute cerebral infarction: a clinical examination scale. Stroke 1989; 20: 864-70.

16. Bamford J, Sandercock PA, Dennis MS, Burn J, Warlow CP. Classification and natural history of clinically identifiable subtypes of brain infarction. Lancet 1991; 337: 1521-6.

17. Lin K, Rapalino O, Law M, Babb JS, Siller KA, Pramanik BK. Accuracy of the Alberta Stroke Program Early CT Score during the First 3 Hours of Middle Cerebral Artery Stroke: Comparison of Noncontrast CT, CT Angiography Source Images, and CT Perfusion. AJNR Am J Neuroradiol 2008; 29: 931-6.

18. Wahlgren N, Ahmed N, Dávalos A, Ford GA, Grond M, Hacke W, et al. Thrombolysis with alteplase for acute ischaemic stroke in the Safe Implementation of Thrombolysis in Stroke-Monitoring Study (SITS-MOST): an observational study. Lancet 2007; 369: 275-82.

19. Hacke W, Kaste M, Fieschi C, von Kummer R, Dávalos A, Meier D, et al. Randomised double-blind placebo-controlled trial of thrombolytic therapy with intravenous alteplase in acute ischaemic stroke (ECASS II). Second European-Australasian Acute Stroke Study Investigators. Lancet 1998; 352(9136):1245-51.

20. Van Swieten JC, Koudstaal PJ, Visser MC, Schouten HJ, van Gijn J. Interobserver agreement for the assessment of handicap in stroke patients. Stroke 1988; 19 (5): 604-7.

21. Adams HP, Bendixen BH, Kappelle LJ, Biller J, Love BB, Gordon DL, et al. Classification of subtype of acute ischemic stroke: definitions for use in a multicenter clinical trial. Stroke 1993; 24: 35-41.

22. Less KR, Bluhmki E, von Kummer R, Brott TG, Toni D, Grotta JC, et al. Time to treatment with intravenous alteplase and outcome in stroke: an updated pooled analysis of ECASS, ATLANTIS, NINDS, and EPITHET trials. Lancet 2010; 375: 1695-703.

23. Saver JL, Fonarow GC, Smith EE, Reeves MJ, Grau-Sepulveda MV, Pan W, et al. Time to Treatment With Intravenous Tissue Plasminogen Activator and Outcome From Acute Ischemic Stroke. JAMA 2013; 309 (23): 2480-8.

24. Jauch EC, Saver JL, Adams HP Jr, Bruno A, Connors JJ, Demaerschalk BM, et al. Guidelines for the early management of patients with acute ischemic stroke: a guideline for healthcare professionals from the American Heart Association/American Stroke Association. Stroke 2013; 44: 870-947.

25. Fonarow GC, Smith EE, Saver JL, Reeves MJ, Bhatt DL, 
Grau-Sepulveda MV, et al. Timeliness of tissue-type plasminogen activator therapy in acute ischemic stroke: patient characteristics, hospital factors, and outcomes associated with door-to-needle times within 60 minutes. Circulation 2011; 123 (7): 750-8.

26. Strbian D, Ahmed N, Wahlgren N, Lees KR, Toni D, Roffe C, et al. Trends in Door-to-Thrombolysis Time in the Safe Implementation of Stroke Thrombolysis Registry: Effect of Center Volume and Duration of Registry Membership. Stroke 2015; 46: 1275-80.

27. Sajobi TT, Menon BK, Wang M, Lawal O, Shuaib A, Williams D, et al. Early Trajectory of Stroke Severity Predicts Long-Term Functional Outcomes in Ischemic Stroke Subjects: Results From the ESCAPE Trial (Endovascular Treatment for Small Core and Anterior Circulation Proximal Occlusion With Emphasis on Minimizing CT to Recanalization Times). Stroke 2017; 48: 105-10.

28. Kerr DM, Fulton RL, Lees KR. Seven-Day NIHSS Is a Sensitive Outcome Measure for Exploratory Clinical Trials in Acute Stroke: Evidence From the Virtual
International Stroke Trials Archive. Stroke 2012; 43: 1401-3.

29. Wardlaw JM, Murray V, Berge E, del Zoppo G, Sandercock P, Lindley RL, et al. Recombinant tissue plasminogen activator for acute ischaemic stroke: an updated systematic review and meta-analysis. The Lancet 2012; 379 (9834): 2364-72.

30. Stroke Unit Trialists'Collaboration. Organised inpatient (stroke unit) care for stroke. Cochrane Database of Systematic Reviews 2007, issue 4. Art. No: CD000197. 2009. DOI: 10.1002/14651858.CD000197.pub2.

31. Guevara C, Bulatova K, Aravena F, Caba S, Monsalve J, Lara $\mathrm{H}$, et al. Trombolisis intravenosa en accidente cerebro vascular isquémico agudo en un hospital público de Chile: Análisis prospectivo de 54 casos. Rev Med Chile 2016; 144: 442-50.

32. Nogales-Gaete J, Núñez L, Arriagada C, Sáez D, Figueroa $\mathrm{T}$, Fernández R, et al. Clinical characterization of 450 patients with cerebrovascular disease admitted to a public hospital during 1997. Rev Med Chile 2000; 128 (11): 1227-36. 